\title{
Los objetos de aprendizaje como conductores para favorecer el aprendizaje autónomo en el campo de la mecánica de fluidos.
}

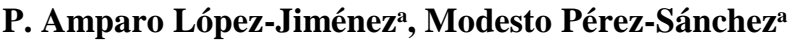

a Departamento de Ingeniería Hidráulica y Medio Ambiente. Universitat Politècnica de València. palopez@upv.es; mopesan1@upv.es

\begin{abstract}
This paper describes the authors' experience in the field of autonomous learning in the areas of fluid mechanics across learning objects. Fluid mechanics is a cross-discipline in the curriculum of engineering students, as it addresses the problems of movement of water and air; and is part of basic materials in the preparation of future engineers as the foundation for their studies installations, hydraulic machinery, energy, environmental matters and dispersion of pollutants, sustainability, etc. In this sense, the main concepts of mechanical fluids are not easy to transmit with the times that are available for classroom theory. The availability of learning objects in this field becomes an important opportunity for students to support their role in the adquisition of the concepts presented here as a complementary tool. The experience of the authors in this field is already long and the results of monitoring and consultation of objects made freely available to the students, very positive.
\end{abstract}

Keywords: learning objects, fluid mechanics, autonomous learning

\section{Resumen}

El presente trabajo describe la experiencia de los autores en el campo del aprendizaje autónomo en materias de mecánica de fluidos a través de objetos de aprendizaje. La mecánica de fluidos es una disciplina muy transversal en los programas curriculares de los alumnos de ingenierías, puesto que aborda los problemas de movimiento de agua y aire y se encuentra formando parte de materias básicas en la preparación de los futuros ingenieros como pilar para sus estudios de instalaciones, maquinaria hidráulica, energía, materias ambientales y de dispersión de contaminantes, materias de sostenibilidad, etc. En este sentido, los conceptos principales de la mecánica de fluidos no son sencillos de transmitir en ocasiones, con los tiempos en los que se dispone para teoría de aula. La disponibilidad de objetos de aprendizaje en este campo, se convierte en una herramienta complementaria importante para que los alumnos puedan apoyarse a su ritmo en la adquisición de los conceptos que aquí se presentan. La experiencia de los autores en este campo es ya larga y los resultados del seguimiento y las consultas de los objetos puestos a libre disposición del alumnado, muy satisfactoria.

Palabras clave: objetos de aprendizaje, mecánica de fluidos, aprendizaje autónomo 


\section{Introducción}

La mecánica de fluidos es una rama dentro de la física de medios continuos que se encarga de estudiar el movimiento en el seno de aquellas sustancias en que siempre que se produce una acción (fuerza tangencial) aparece una deformación (campo de velocidades). En este sentido, el análisis de esta materia en el marco de los estudios de ingeniería se encuentra en los primeros cursos y de forma natural, como una continuación de los estudios de física.

A su vez, la mecánica de fluidos es la base teórica de la ingeniería hidráulica: el cómo se ha llevado a cabo a lo largo del tiempo implementado en aplicaciones prácticas ingenieriles, estos conocimientos de la física, y se encuentra como base en muchas materias curriculares de los estudiantes de todos los grados de ingeniería en cursos superiores. Por ello se hace necesario que el alumno adquiera y conozca en profundidad los conceptos físicos involucrados con la mecánica de fluidos para aplicarlos en cursos superiores y en el desarrollo de su vida profesional.

En este sentido, el aprendizaje del alumno debe valerse de todas las estrategias posibles para que alcance un grado importante de efectividad, máxime teniendo en cuenta que la docencia universitaria se encuentra en proceso de cambio no sólo como consecuencia del cambio de planes de estudio a la convergencia europea sino al desarrollo de las tecnologías de información y comunicación (Álvarez et al., 2005). Entre ellas se encuentra su propia capacidad para asimilar los conocimientos a través de un aprendizaje autónomo que, con las herramientas adecuadas, le permita conocer a su propio ritmo las ideas fundamentales que deben asentarse en una materia básica como la que nos ocupa. Bajo la experiencia de los autores, una de las herramientas más potentes es la puesta a disposición del alumnado de los objetos de aprendizaje, con el objetivo de potenciar el aprendizaje autónomo para facilitar la adquisición de los conocimientos necesarios (Marzano, 1991)

Los objetos de aprendizaje son unidades mínimas de aprendizaje, en formato digital, que pueden ser reutilizadas y secuenciadas. En estos videos cortos, de entre cinco y diez minutos de duración, el profesor desarrolla una idea a través de una explicación y una presentación en la que a su vez, pueden incluirse modelos numéricos, otros vídeos o enlaces diversos. Estos objetos de aprendizaje están estructurados de tal forma que estructuran algunas de las unidades didácticas de la asignatura de Mecánica de Fluidos, permitiendo mejorar el alcance de los resultados de aprendizaje. De igual modo, al alumno le puede permitir repasar contenidos explicados en clases presenciales así como la realización de tareas autónomonas o grupales propuestas. En el campo de la mecánica de fluidos los conceptos a desarrollar son prácticamente interminables puesto que desde la propia definición de los fluidos hasta los conceptos más complejos de la turbulencia, todos ellos permiten una presentación bajo este tipo de elementos de comunicación con el alumno a través de docencia asíncrona como son los objetos de aprendizaje. Así, pues, el que los alumnos dispongan de estas grabaciones, donde con todo el detalle que corresponda y haciendo llamamiento a otra bibliografía o enlaces, puedan asimilar estos conceptos, se convierte en una herramienta complementaria muy importante para sus estudios y ha dado muy buenos resultados en los últimos tiempos, al proponer de forma abierta estos objetos a toda la comunidad universitaria global.

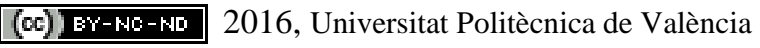


El uso de las nuevas tecnologías para un aprendizaje autónomo es conocido desde hace algunas décadas (Garrison, 1998) reconociendo su gran potencia. En el caso particular de la mecánica de fluidos, dado el grado de complejidad de los aspectos tratados la importancia de estas materias como una base avanzada de los conocimientos de física, son los alumnos de primeros cursos de grado, pero no principiantes, los que más han hecho uso de los mismos. Se han presentado los objetos de aprendizaje como propuesta de formación autónoma cuando se han impartido los temarios en aula (como elementos complementarios que aumenten el índice de retención de los conceptos (Rue, 2009)); o de forma abierta cuando se han preparado cursos específicos o a disposición del alumnado en general. La apertura de estos materiales a foros internacionales enriquece el contenido y las consultas de los alumnos. Las experiencias en este campo se han documentado en el marco de estudios de últimos ciclos en la Universidad española ya en la última década (Onrubia et al, 2006).

Sin embargo, por el hecho de proponer una estrategia en que el alumno potencie su propio aprendizaje autónomo, el profesor no deja de tener un rol fundamental. Por el contrario, el profesor que prepara y presenta los objetos juega a continuación un papel de "tutor a distancia" de alumnos a los que conoce o no conoce, que consultan los materiales de forma concreta, jugando un papel de apoyo temporal que facilite a los alumnos, en este espacio virtual, que éstos ejecuten su nivel justo de aprendizaje, más allá de la competencia corriente de habilidades que poseen y que reciben en su docencia presencial (Pagano, 2007). Para hacer llegar las competencias científicas a los estudiantes y que ellos puedan plasmarlo en la resolución de problemas que exigen sus diferentes competencias, se hace muy interesante el uso de tecnologías de información y simulaciones gráficas o presentaciones como las aquí descritas, que permitan a los alumnos asimilar los conocimientos en repetidas ocasiones y a un ritmo mucho más pausado de lo que la clase magistral particularmente permite en cada grado (Edith y Alvarado, 2014).

\section{Objetivos}

Lograr que los alumnos de ingeniería aprendan sobre la disciplina de mecánica de fluidos, involucra muchos aspectos de la física, desde el concepto de medios continuos hasta la comprensión de fenómenos complejos como la turbulencia y la capa límite. En este sentido se han desarrollado breves objetos de aprendizaje para atomizar los conceptos y objetivos docentes y proponer a los alumnos ideas fundamentales que les ayuden, bajo su propio ritmo, a adquirir dichos conceptos. Hasta el momento, se describen a continuación los objetos desarrollados más en la línea de la presente contribución, con sus objetivos docentes:

- La hidráulica y la mecánica de fluidos: historia, ciencia y técnica. La Hidráulica y la mecánica de fluidos son dos disciplinas que han ido de la mano, a pesar de que la primera es eminentemente práctica y la segunda teórica. El movimiento del agua y otros fluidos se ha estudiado casi desde el principio de la humanidad y los avances en el estudio del movimiento del agua particularmente han interesado a ingenieros desde las civilizaciones antiguas. En este caso, se presentan los científicos e ingenieros más eminentes en ambos campos, analizando las aportaciones más importantes de cada uno de ellos.

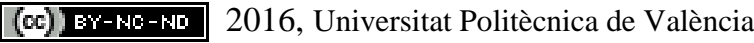

Congreso In-Red (2016) 
- Propiedades de los fluidos. Los fluidos tienen una dinámica compleja. Son un medio continuo que se encuentra en todo lo que nos rodea, por lo que el conocimiento de su comportamiento en reposo y en movimiento es extraordinariamente importante en todos los ámbitos que nos afectan. Es fundamental caracterizar bien la naturaleza de cada fluido para comenzar un estudio del mismo, bien sea éste un análisis experimental o computacional. En el presente objeto de aprendizaje se introducen las propiedades principales de los fluidos que definen esta caracterización, las magnitudes y unidades que las representan; así como la clasificación de los mismos atendiendo a estas propiedades definitivas.

- La estática de fluidos. La estática de fluidos es la parte de la física que estudia el reposo de los fluidos. Cuando los fluidos no se mueven, se experimentan en el seno de los mismos unos efectos debidos a la presión existente en cada punto, que a pesar del reposo de los mismos, siempre generan fuerzas en los elementos externos que los contienen. El análisis de las presiones, acciones sobre superficies, fuerzas y empuje sobre los cuerpos sumergidos es lo que se abordará en el presente objeto de aprendizaje.

- La ecuación de Bernoulli. La aplicación de la ecuación de conservación de la energía a los fluidos en movimiento es compleja cuando se tienen en cuenta todas las formas de energía. Sin embargo, la particularización de esta ecuación en régimen permanente cuando se tienen en cuenta solamente las manifestaciones de energía mecánica en los fluidos, es un concepto mucho más simple, representado en la Ecuación de Bernoulli. Se presenta en este objeto, esta ecuación que relaciona todas las formas de energía cinética y potencial a lo largo de una línea de corriente en un fluido y se analizan las posibles implicaciones prácticas de esta ecuación como acercamiento al análisis energético en este tipo de flujos.

- Introducción a la cinemática de fluidos, conceptos fundamentales. La cinemática de fluidos representa a los fluidos en movimiento, de manera que hay que analizar los aspectos de velocidad de dichos movimientos. Se plantea en este objeto de aprendizaje la clasificación de los flujos con objeto de reconocer las características de los mismos en base a los aspectos del movimiento. Se presenta asimismo los diferentes enfoques en cuanto al análisis de coordenadas de los mismos así como los conceptos básicos de trayectoria y línea de corriente, tan ligados al análisis cinemático de la mecánica de fluidos

- Introducción al problema de la turbulencia. La mecánica de fluidos puede ser representada mediante un conjunto de ecuaciones fundamentales que, una vez resueltas, proporcionan las velocidades, presiones y fuerzas en todos los puntos de estudio. Sin embargo, la naturaleza de la mecánica del medio continuo al que pertenecen los fluidos y su movimiento caótico, hacen que deban ser consideradas las condiciones de turbulencia: las velocidades reales en cada momento son la suma de una componente media, más una componente fluctuante, que complica enormemente las ecuaciones de conservación que deben ser resueltas. En el presente objeto de aprendizaje, se presenta el problema de la turbulencia, las complicaciones que supone en el modelo y las posibilidades de resolución que ofrece.

- Ecuaciones fundamentales de la fluidodinámica computacional. La mecánica de fluidos es una disciplina de la física de gran interés, puesto que en todas las actividades de nuestra vida diaria nos encontramos rodeados de fluidos. En este sentido, el conocimiento de las velocidades, presiones y fuerzas que realizan los mismos en su entorno debe ser abordado resolviendo un conjunto de ecuaciones que representan este comportamiento, complejo de por sí. El presente objeto de aprendizaje presenta estas ecuaciones y las formas en las que las posibilidades de la

(cc) EY-NC-ND 2016, Universitat Politècnica de València 
computación actual nos permiten resolverlas y encontrar las magnitudes que representan su comportamiento en todos los entornos considerados

- La fluidodinámica computacional: casos de aplicación. La fluidodinámica computacional (CFD por sus siglas en inglés: Computational Fluid Mechanics) permite resolver las ecuaciones de la mecánica de fluidos mediante técnicas numéricas, hoy en día abordadas mediante ordenador. La resolución de estas ecuaciones es una tarea compleja y se suelen utilizar códigos desarrollados expresamente para esa labor. En el presente objeto de aprendizaje, se presentan algunos de los casos concretos en el campo científico-técnico en que tienen cabida estas estrategias de modelación y que pueden ser de gran utilidad.

\section{Desarrollo de la innovación}

La innovación en este campo se entiende como la construcción de ideas atomizadas que el alumno adquiere a su propio ritmo en torno a problemáticas significativas de la mecánica de fluidos que se encuentra en la base de muchas titulaciones de ingeniería en el marco del nuevo Espacio Europeo de Educación Superior. La producción de objetos así, podrán ser orientados al desarrollo de competencias que tengan un planteamiento lo suficientemente abierto como para ser contextualizados por alumnos procedentes de diversidad de ámbitos y favorece el aprendizaje autónomo basado en las nuevas tecnologías a través de los vídeos dejados a disposición de los alumnos.

En este sentido, se considera que se han conseguido los siguientes hitos:

- Se motiva al alumno a organizar su tiempo y tareas como apoyo a sus clases teóricas. Los objetos se encuentran a disposición del alumno sin obligación de usarse. Ello hace que el aprendizaje se convierta en una actividad flexible, en la que el estudiante marca su propio ritmo y puede profundizar en aquellos temas que hayan despertado más su atención o su interés en cada asignatura entorno a la mecánica de fluidos.

- $\quad$ Se potencia una comunicación bidireccional entre el alumno y el profesor, a través del foro de cada asignatura en que pueden incrustarse los objetos, de manera que favorece las tutorías a distancia, apoyado sobre los diferentes elementos de la presentación que se encuentran en los vídeos de los objetos

- Se potencia el uso de nuevas tecnologías a través de Internet, como recurso facilitador del aprendizaje. Ello hace que las nuevas tecnologías se conviertan en una herramienta para la el acceso al conocimiento y la autonomía del alumnado en cuanto a su aprendizaje, que le permite acceder a los conceptos en cualquier momento desde su ordenador.

- Permite al profesor establecer un vínculo más allá de las fronteras espaciotemporales del curso con el alumno, de forma que pueda resultar de apoyo en las materias de mecánica de fluidos y aquellas que se apoyan en esta disciplina.

- $\quad$ Pueden proponerse y/o desarrollarse actividades (e-portafolio, tareas, test de autoevaluación...) con el objetivo que estos objetos de aprendizaje favorezcan el aprendizaje

(c)) EY-NC-ND 2016, Universitat Politècnica de València

Congreso In-Red (2016) 
autónomo del alumno, facilitando de esta forma alcanzar los resultados de aprendizaje de la asignatura.

El seguimiento de estos objetos también presenta puntos débiles que los alumnos tienen que vencer: el aprendizaje autónomo implica que el propio alumno debe marcar sus tiempos, con lo que el grado de aprovechamiento depende de la voluntad del estudiante. Deben tenerse instrumentos para conectarse a los vídeos o acceder a otros programas computacionales descritos en los mismos, o la complejidad de los contenidos, hacen que el alumno no se motive, y no considere interesante la implementación de los resultados de este aprendizaje. Sin embargo, estas dificultades se vencen en tanto que los objetos que aquí se proponen sirven como elemento complementario a una formación en aula y el alumno siempre debe encontrar al profesor para contestar sus dudas, proponer ejemplos o problemas o comunicarse con el alumno de forma directa o a través del correo electrónico, en una sociedad cada vez más comunicada. El desarrollo de los objetos de aprendizaje que se presentan en este documento, pueden constituir el módulo de aprendizaje básico de esta asignatura a partir del cual se podrán desarrollar nuevos objetos más específicos que complementen los contenidos de cada una de las unidades temáticas que componen la asignatura.

\section{Resultados}

Los resultados del seguimiento de los objetos presentados han sido muy satisfactorios a tenor de los correos electrónicos que se han contestado en atención a los mismos de forma complementaria al aprendizaje de los alumnos.

Tabla 1. Seguimiento de los diferentes objetos de aprendizaje descritos.

\begin{tabular}{|c|c|c|}
\hline Objeto & Visualizaciones & $\begin{array}{c}\text { País con más } \\
\text { visualizaciones }\end{array}$ \\
\hline $\begin{array}{c}\text { La hidráulica y la mecánica de fluidos: historia, } \\
\text { ciencia y técnica }\end{array}$ & 141 & $\begin{array}{l}\text { Estados Unidos } \\
\text { (19) }\end{array}$ \\
\hline Propiedades de los fluidos & 1035 & México (85) \\
\hline La estática de fluidos & 76 & España (14) \\
\hline La ecuación de Bernoulli & 76 & España (13) \\
\hline $\begin{array}{c}\text { Introducción a la cinemática de fluidos, } \\
\text { conceptos fundamentales }\end{array}$ & 90 & $\begin{array}{l}\text { Estados Unidos } \\
\qquad(15)\end{array}$ \\
\hline Introducción al problema de la turbulencia & 153 & España (17) \\
\hline $\begin{array}{l}\text { Ecuaciones fundamentales de la fluidodinámica } \\
\text { computacional }\end{array}$ & 280 & $\begin{array}{l}\text { Estados Unidos } \\
\text { (18) }\end{array}$ \\
\hline $\begin{array}{l}\text { La fluidodinámica computacional: casos de } \\
\text { aplicación. }\end{array}$ & 209 & España (24) \\
\hline
\end{tabular}

Siempre es más sencillo cuantificar los resultados cuando se tienen estadísticas del seguimiento que, aunque no indiquen el grado de profundidad al que ha podido llegarse en el aprendizaje autónomo, sí que dan idea del impacto que pueden haber tenido en la

(c)) BY-NC-ND 2016, Universitat Politècnica de València 
comunidad universitaria a la que se ha ofrecido. Así pues, las estadísticas del seguimiento de los objetos destacados son las que se muestran en la Tabla 1, en dicha tabla se encuentran recogidas las visualizaciones de los alumnos que han cursado la asignatura.

Como puede observarse, el seguimiento de los objetos ha sido importante, particularmente entre los estudiantes de las titulaciones españolas y de otros países, puesto que el conocimiento de estas materias se convierte en pilar fundamental para otras. Cabe destacar que el objeto que tiene un mayor seguimiento es uno de los más básicos sobre las propiedades de los medios continuos para considerarse fluidos. Este tema es tan fundamental como poco abordado en la literatura docente complementaria, lo que lo convierte en un concepto que requiere la máxima atención por parte de los alumnos y ha recibido el máximo seguimiento.

\section{Conclusiones}

La integración de objetos de aprendizaje en el marco de las disciplinas relacionadas con la mecánica de fluidos proporciona un nexo interesante para relacionar progresivamente los medios tecnológicos y el aprendizaje autónomo, permite sintetizar y atomizar los conceptos, y favorece una mayor reflexión autónoma por parte del alumno, fundamental para asentar los conocimientos en el largo plazo.

El seguimiento de los objetos descritos por parte de alumnos de todo el mundo, favorecido por la transcripción y traducción escrita de los textos dictados, ha favorecido una mayor comprensión de la situación y motivación, según indican alumnos que han seguido los objetos. Se advierte, de esta manera, un recurso expresivo eficaz para una integración pedagógica amplia de los contenidos presentados relativos a la mecánica de fluidos en un sentido amplio intercalada en los curricula de diferentes titulaciones.

La elaboración de material que propicie el aprendizaje autónomo en materia de mecánica de fluidos debe estar basada en secuencias narrativas breve y visual, ordenadas e interesantes, para los alumnos de forma que sea lo más motivadora posible. Para ello los profesores deben sentirse implicados completamente para proponer objetos atractivos que a la vez sean buenos transmisores de los conceptos fundamentales. Los buenos resultados basados en las estadísticas del uso de los objetos, avalan el interés de los mismos a lo largo del tiempo que llevan a disposición de todo el alumnado de habla castellana; y por extensión a los de habla inglesa, para los que se han traducido algunos de ellos.

La utilización de algunos objetos de aprendizaje presentados en este documento han servido de base para desarrollar actividades que potencian el aprendizaje permanente del alumno a través de las diferentes alternativas que nos presenta la plataforma Poliformat (es una herramienta de "campus virtual" basada en el proyecto "Sakai") que motiven al alumno a desarrollar el trabajo no presencial necesario para alcanzar los resultados de aprendizaje definidos en la guía docente de las diferentes asignaturas.

(cc)) BY-NC-ND 2016, Universitat Politècnica de València

Congreso In-Red (2016) 


\section{Referencias}

Álvarez, B.; González, C. y García, N. (2007). La motivación y los métodos de evaluación como variables fundamentales para estimular el aprendizaje autónomo. Red $U$. Revista de Docencia Universitaria, Número 2. pp 1-12

Edith Granero, A.; Garcia Alvarado, R. (2014). Aprendizaje temprano de arquitectura sustentable mediante vistas interiores graduadas. Revista Hábitat Sustentable. V4. N1. Junio de 2014. pp 14-24.

GARRISON, D. R. (1998). Andragogy, learner-centeredness, and the educational transaction at a distance. Journal of Distance Education, 3- (2), 123-127.

LÓPEZ JIMÉNEZ, P.A. La hidráulica y la mecánica de fluidos: historia, ciencia y técnica. Consultado en Internet en marzo de 2016 en: https://polimedia.upv.es/visor/?id=ae01c40aaad3-e94f-ad0d-7e126990c3b2

LÓPEZ JIMÉNEZ, P.A. Propiedades de los fluidos. Consultado en Internet en marzo de 2016 en: https://polimedia.upv.es/visor/?id=e2b2fd7c-9198-914b-86a1-d76fe39a5ca8

LÓPEZ JIMÉNEZ, P.A. La estática de fluidos. Consultado en Internet en marzo de 2016 en: https://polimedia.upv.es/visor/?id=920e987c-7f84-9e4a-a7ca-f332446d9526

LÓPEZ JiMÉNEZ, P.A. La ecuación de Bernoulli. Consultado en Internet en marzo de 2016 en: https://polimedia.upv.es/visor/?id=4a4976d6-7244-694b-8f5a-9a9351a5f1e0

LÓPEZ JiMÉNEZ, P.A. Introduccion a la cinemática de fluidos, conceptos fundamentales. Consultado en Internet en marzo de 2016

LÓPEZ JIMÉNEZ, P.A. Introduccion al problema de la turbulencia. Consultado en Internet en marzo de 2016 en: $\quad$ https://polimedia.upv.es/visor/?id=876d5400-53a1-254f-896a-

eb73c5d6c65e

LÓPEZ JiMÉNEZ, P.A. La fluidodinámica computacional: casos de aplicación.. Consultado en Internet en marzo de $2016 \mathrm{en:} \quad$ https://polimedia.upv.es/visor/?id=37b4bc10-17e79947-ba6a-ff5611c7fbc6

MARZANO, R. J. (1991). Creating an educational paradigm centred on learning through teacher-directed, naturalistic inquiry. En L. Idol y B. F. Jones (Eds.), Educational values and cognitive instruction. Implication for reform (411-442). Hillsdale:Erlbaum.

Onrubia, J., Bustos, A., Engel, A.; Segués , T. (2006). Usos de una herramienta de comunicación asíncrona para la innovación docente en contextos universitarios. IV Congreso Internacional de Docencia Universitaria i Innovació. Barcelona . (España).

PAGANO, C.M. (2007). Los tutores en la educación a distancia. Un aporte teórico.» [artículo en línea]. Revista de Universidad y Sociedad del Conocimiento (RUSC). Vol. 4, n. ${ }^{\circ}$ 2. UOC. [Consultada en Abril de 2015 ]. 〈http://www.uoc.edu/rusc/4/2/dt/esp/pagano.pdf>. ISSN 1698-580X.

RUÉ, J. (2009). "El aprendizaje autónomo en educación superior”. Madrid. Narcea Ediciones.

(c)) BY-NC-ND 2016, Universitat Politècnica de València 